\title{
TOPOLOGICAL GROUPS AND GENERALIZED MANIFOLDS
}

\author{
EDWARD G. BEGLE
}

In a recent paper [4],1 Montgomery showed that in a locally euclidean 3-dimensional group, any 2-dimensional closed subgroup is also locally euclidean. In this note we prove an analogous result for higher dimensions and more general spaces.

THEOREM. Let $G$ be a locally compact space which is both a topological group and an n-dimensional orientable generalized manifold. Let $H$ be a closed connected $(n-1)$-dimensional subgroup. Then, if $H$ carries a nonbounding $(n-1)$-cycle, $H$ is also an orientable generalized manifold.

The terminology used in the statement of this theorem, and in what follows, is that of our two previous papers on generalized manifolds $[1,2]$, and we assume that the reader is familiar with them.

We make, however, one change. We find it convenient to define infinite cycles in the following way: We add to $G$ an ideal point, $g^{+}$, taking as neighborhoods of $g^{+}$those open subsets of $G$ whose closures are not compact. Then $G^{+}=G \cup g^{+}$is compact. Now an infinite cycle of $G$ is defined to be a relative cycle of $G^{+} \bmod g^{+}$. That this definition of infinite cycles is equivalent to the one used in [2] follows from Theorem 1.1 of [2].

LEMMA 1. Given any neighborhood $M$ of the unit element $e$ of $G$, there is a neighborhood $N$ of $E$ such that for any infinite cycle $\Gamma^{k}$ on $H, 0 \leqq k$ $\leqq n-1$, and for any $g \in N, \Gamma^{k} \sim g \cdot \Gamma^{k}$ on $M \cdot H$.

Proof. Let $M_{n-1} \subset M$ have a compact closure. Choose a sequence

$$
M_{n-1} \supset N_{n-1} \supset M_{n-2} \supset \cdots \supset M_{0} \supset N_{0}
$$

such that $N_{i}$ is obtained from $M_{i}$ by the local connectedness of $G$ in dimension $i$, and such that $M_{i} \cdot M_{i} \subset N_{i+1}$. Finally let $N$ be such that $N \cdot N \subset N_{0}$.

Now let $g \in N$. To show that $\Gamma^{k} \sim g \cdot \Gamma^{k}$ on $M \cdot H$, it is sufficient to show that the coordinates of these cycles on the nerve of any covering $U$ of $G$ are homologous on $(M \cdot H)^{+}$. To this end, given a covering $U$, choose $U^{\prime}<^{*} U$. Let $X$ be the complement of the union of those sets of $U^{\prime}$ which contain $g^{+}$. Then $X$ is a compact set. Let $X_{1}=\bar{M}_{0} \cdot X$ and $X_{i}=\bar{M}_{i-1} \cdot X_{i-1}$. Each $X_{i}$ is a compact set.

Received by the editors December 10, 1947.

1 Numbers in brackets refer to the bibliography at the end of the paper. 
A finite number of translations of $N_{n-1}$ cover $X_{n}$, say

$$
g_{1, n-1} \cdot N_{n-1}, g_{2, n-1} \cdot N_{n-1}, \cdots, g_{k, n-1} \cdot N_{n-1} \text {. }
$$

For each $i$, let $U_{i, n-1}$ be a refinement of $U^{\prime}$ such that any $(n-1)$ cycle on $U_{i, n-1}$ in $g_{i, n-1} \cdot N_{n-1}$ has its projection to $U^{\prime}$ bounding in $g_{i, n-1} \cdot M_{n-1}$. Let $U_{n-1}$ be a common refinement of these coverings.

Next, a finite number of translations of $N_{n-2}$ cover $X_{n-1}$. From these we obtain a refinement $U_{n-2}$ of $U_{n-1}$ by the procedure above, this time using the local connectedness of $G$ in dimension $n-2$. Proceeding in this fashion for another $n-2$ steps we arrive at a covering $U_{0}$.

Let $\Gamma_{0}^{k}$ and $g \cdot \Gamma_{0}^{k}$ be the coordinates of $\Gamma^{k}$ and $g \cdot \Gamma^{k}$ on $U_{0}$. We assert that $\pi \Gamma_{0}^{k}$ and $\pi g \cdot \Gamma_{0}^{k}$ are homologous on $U$ on $(M \cdot H)^{+}$, where $\pi$ is the projection from $U_{0}$ to $U$. Let $\Delta$ be the cartesian product of $\left|\Gamma_{0}^{\boldsymbol{k}}\right|$ with a unit segment, subdivided simplicially in such a way that all the vertices of $\Delta$ are in the base, $\Delta_{0}=\left|\Gamma_{0}^{k}\right| \times 0$, and in the top, $\Delta_{1}$ $=\left|\Gamma_{0}^{k}\right| \times 1$. Let $\bar{\Delta}$ be the closed subcomplex of $\Delta$ generated by those simplexes of $\Gamma_{0}^{k}$ which are on $X$. We define a partial realization $\tau^{\prime}$ of $\Delta$ on $U_{0}$ by letting $\tau^{\prime} \sigma=\sigma$ if $\sigma \in \Delta_{0}$ and $\tau^{\prime} \sigma=g \cdot \sigma$ if $\sigma \in \Delta_{1}$.

$\tau^{\prime}$ induces a partial realization $\bar{\tau}^{\prime}$ of $\bar{\Delta}$ on $U_{0}$. In view of the choices of the coverings made above, the usual argument shows that there is a full realization $\bar{\tau}$ of $\bar{\Delta}$ on $U^{\prime}$, where, if $\pi_{0}$ is the projection from $U_{0}$ to $U^{\prime}, \bar{\tau}=\pi_{0} \bar{\tau}^{\prime}$ whenever the latter is defined. Also, $\bar{\tau} \bar{\Delta}$ is on $M_{n-1} \cdot H$.

We can now define a full realization of $\Delta$ on $U$ in the following fashion. The projection $\pi_{0}$ can be so chosen that a vertex of $U_{0}$ not on $X$ is projected into a vertex of $U^{\prime}$ which contains $g^{+}$. Since $U^{\prime}<^{*} U$, a projection $\pi$ of $U^{\prime}$ to $U$ can be so chosen that any simplex of $U_{0}$ which has a vertex not on $X$ is projected by $\pi \pi_{0}$ into the simplex of $U$ consisting of those vertices of $U$ which contain $g^{+}$. Any cycle in this simplex bounds in this simplex, so $\pi \pi_{0} \tau^{\prime}(\Delta-\bar{\Delta})$ can be filled in to make a full realization of $\Delta-\bar{\Delta}$ on $U$, and this together with $\pi \bar{\tau} \bar{\Delta}$ makes a full realization $\tau$ of $\Delta$ on $U$. Since $\Gamma_{0}^{k} \times 0 \sim \Gamma_{0}^{k} \times 1$ on $\Delta$, $\tau\left(\Gamma_{0}^{k} \times 0\right) \sim \tau\left(\Gamma_{0}^{k} \times 1\right)$ on $U$. But $\tau\left(\Gamma_{0}^{k} \times 0\right)=\pi \pi_{0} \Gamma_{0}^{k}$ and $\tau\left(\Gamma_{0}^{k} \times 1\right)=\pi \pi_{0} g$ $\cdot \Gamma_{0}^{k}$. Since it is easily seen from the construction that this homology takes place on $(M \cdot H)^{+}$, the proof is complete.

Clearly the same proof suffices for the following lemma.

LEMMA 2. Given any neighborhood $M$ of $e$ in $G$, there is a neighborhood $N$ of e such that for any closed subset $X$ of $H$ and any cycle $\Gamma^{k}$ of $H$ mod $X, \Gamma^{k} \sim g \cdot \Gamma^{k}$ in $M \cdot H \bmod M \cdot X$, whenever $g \in N$.

LEMMA 3. If $D$ is an open connected subset of $G$, then any two points 
of $D$ lie on a compact connected subset of $D$.

Proof. Since $G$ is $l c^{0}$, any neighborhood $O$ of a point $d$ of $D$ contains a neighborhood $W$ of $d$ with $W \subset D$ such that any point $w \in W$ lies, together with $d$, on a compact continuum in $O \cap D$. Now let $D_{1}$ be the set of all points of $D$ which can be joined to a fixed point $d_{1} \in D$ by compact continua. Then, by the above, $D_{1}$ is both open and closed in $D$. Hence, since $D$ is connected, $D_{1}$ is all of $D$.

LEMma 4. If $O$ is a neighborhood of e such that $\mathcal{C}(O \cdot H)$ (where $C$ means closure) is not all of $G$, then $O \cdot H-H$ has at least two components.

Proof. Let $g$ be a point of $G-\mathcal{C}(O \cdot H)$, and let $K$ be a compact connected set which contains both $e$ and $g$. Let $N$ be a neighborhood of $e$ in $O$, chosen by Lemma 1. A finite number of translations of $N$ cover $K$, and from these we may choose a sequence

$$
e \in N, N_{1}, N_{2}, \cdots, N_{k} \ni g
$$

where $N_{i}=g_{i} \cdot N$ and such that $N_{i} \cap N_{i+1} \neq 0$. Let $\bar{g}_{i}$ be a point of $N_{i} \cap N_{i+1}$. Now, $\bar{g}_{i-1} \in g_{i} \cdot N$, so $g_{i}^{-1} \cdot \bar{g}_{i-1} \in N$. Hence, by Lemma 1 ,

$$
\Gamma^{n-1} \sim g_{i}^{-1} \cdot \bar{g}_{i-1} \cdot \Gamma^{n-1}
$$

where $\Gamma^{n-1}$ is a nonbounding cycle on $H$. Therefore,

$$
g_{i} \cdot \Gamma^{n-1} \sim \bar{g}_{i-1} \cdot \Gamma^{n-1} .
$$

Similarly, $\bar{g}_{i} \in g_{i} \cdot N$, and

$$
g_{i} \cdot \Gamma^{n-1} \sim \bar{g}_{i} \cdot \Gamma^{n-1} .
$$

Thus, we have

$$
\Gamma^{n-1} \sim g \cdot \Gamma^{n-1} .
$$

Now $\Gamma^{n-1}-g \cdot \Gamma^{n-1}$ is a cycle of $H \cup(G-O \cdot H)$ and $\Gamma^{n-1}-g \cdot \Gamma^{n-1} \sim 0$ in $G$. Hence $[3$, p. $227(14.2)]$ there is in $G$ a cycle $\Gamma^{n} \bmod (H \cup(G$ $-O \cdot H)$ ) such that $F \Gamma^{n}=\Gamma^{n-1}-g \cdot \Gamma^{n-1}$. Let $\Gamma^{n}$ be the fundamental $n$-cycle of $G$, and let $\Gamma_{1}^{n}$ be the part of $\Gamma^{n}$ on $G-(O \cdot H-H)$. Let $\bar{\Gamma}_{2}^{n}=\bar{\Gamma}^{n}-\Gamma_{1}^{n}$.

In a neighborhood of any point of $O \cdot H-H, \Gamma^{n}$ is homologous to some multiple of $\Gamma^{n}$. If we assume that $O \cdot H-H$ is connected, then (cf. $[1$, p. 569]) this multiple, is the same for all points of $O \cdot H-H$, that is, $r \Gamma^{n}=\Gamma_{2}^{n}$.

By definition, $\Gamma_{1}^{n}$ is on $H \cup(G-O \cdot H)$. Since $H$ and $G-O \cdot H$ are closed and disjoint, and since $\operatorname{dim} H<n, \Gamma_{1}^{n}$ must be on $G-O \cdot H$, so $F \bar{\Gamma}_{1}^{n}$ is also on $G-O \cdot H$. 
But, from $0=F \bar{\Gamma}^{n}=F\left(\Gamma_{1}^{n}+\Gamma_{2}^{n}\right)$, we have

$$
\begin{aligned}
F \bar{\Gamma}_{1}^{n} & =-F\left(\bar{\Gamma}_{2}^{n}\right)=-F\left(r \Gamma^{n}\right) \\
& =-r\left(\Gamma^{n-1}-g \cdot \Gamma^{n-1}\right) .
\end{aligned}
$$

This is not on $G-O \cdot H$, since $\Gamma^{n-1}$ is on $H$. Thus, the assumption that $O \cdot H-H$ has only one component leads to a contradiction.

We now choose a fixed connected neighborhood of $e$, satisfying the condition of Lemma 4, and denote by $J$ the product of $H$ by this neighborhood. We note that $J$ is a connected generalized $n$-manifold. It is not a group, but for any two elements of $J$ which are close enough to $H$, their product in $G$ is in $J$.

LEMMA 5. $H$ is the boundary of each domain of $J-H$.

Proof. Let $D$ be any component of $J-H$. Since $J$ is $l c^{0}, D$ is open. Some point $h \in H$ is a limit point of $D$, or else $J$ would not be connected. Let $O$ be a neighborhood of $e$. Then $h \cdot O$ contains a point $d \in D$, that is, $h \cdot o=d$. Now $h^{-1} \cdot d=o \in O$. But $h^{-1} \cdot d$ is also in $D$. For $o \notin H$, and, since $H$ is connected, $H \cdot o$ lies in one component of $J-H$. Since $h \cdot o=d$ is in $D, H \cdot o$ lies in $D$, and consequently, $e \cdot o=o$ is in $D$. Therefore $e$ is a limit point of $D$. Similarly, if $\bar{h}$ is any other point of $H$, then the neighborhood $\bar{h} \cdot O$ contains $\bar{h} \cdot O$ which is in $H \cdot O$ and therefore in $D$. Thus, $\bar{h}$ is a limit point of $D$, which proves the lemma.

LEMMA 6. $J-H$ has just two components.

Proof. ${ }^{2}$ By Lemma 2, it is enough to show that $H$ does not have three complementary domains. Suppose there were three, $D_{0}, D_{1}$ and $D_{2}$. Let $p_{1}, p_{2}$ be points in $D_{1}$ and $D_{2}$ respectively, and let $Y_{1}, Y_{2}$ be neighborhoods of $p_{1}, p_{2}$ such that $\bar{Y}_{i}$ is compact and is in $D_{i}$.

$\boldsymbol{\gamma}^{0}=p_{1}-p_{2}$ is a compact 0 -dimensional cycle in $Y=Y_{1} \cup Y_{2} \cdot \gamma^{0}$ not $\sim 0$ in $J-H$, since $p_{1}$ and $p_{2}$ are in different components. But for any point $d_{0} \in D_{0}, \gamma^{0} \sim 0$ in $J-d_{0} \cdot H$. For let $O$ be a neighborhood of $e$ not meeting $d_{0} \cdot H$, which is in $D_{0}$, and let $O^{\prime}$ be chosen so that every compact 0 -cycle in $O^{\prime}$ bounds in $O$. Choose $d_{1} \in O^{\prime} \cap D_{1}$ and $d_{2} \in O^{\prime}$ $\cap D_{2}$. Then $d_{1} \sim d_{2}$ in $O$. By Lemma $3, p_{1} \sim d_{1}$ in $D_{1}, p_{2} \sim d_{2}$ in $D_{2}$. Hence, $p_{1} \sim p_{2}$ in $D_{1} \cup D_{2} \cup O$, which does not meet $d_{0} \cdot H$.

Now, by Lemma 5.2 of [2], there is a compact cocycle $\gamma_{n}$ in $Y$ such that $\left(\bar{\Gamma}^{n} \cdot \gamma_{n}\right) \sim \gamma^{0}$ in $J-H$, where $\bar{\Gamma}^{n}$ is the fundamental $n$-cycle of $J$, and such that $\gamma_{n} \sim 0$ in $J-d_{0} \cdot H$, for any $d_{0} \in D_{0}$. Since $\gamma_{n}$ is a compact cocycle of $D_{1} \cup D_{2}$, there is an infinite $n$-cycle $\Gamma^{n}$ of $D_{1} \cup D_{2}$

${ }^{2}$ The main outline of this proof, and to some extent that of Lemma 7, is derived from Wilder $[6,7]$. 
such that $\mathrm{KI}\left(\Gamma^{n} \cdot \gamma_{n}\right)=1$. Let $\Gamma^{n-1}=F \Gamma^{n}$, so that $\Gamma^{n-1}$ is an infinite cycle of $H$.

We now choose a neighborhood $M$ of $e$ which does not meet $\bar{Y}$, and a neighborhood $N$ satisfying the conditions of Lemma 1. Let $d_{0} \in D_{0} \cap N$. Then $\Gamma^{n-1} \sim d_{0} \cdot \Gamma^{n-1}$ in $M \cdot H$. Let $\Gamma^{n}=\left\{\Gamma_{\zeta}^{n}\right\}$ and let the chains involved in the homology $\Gamma^{n-1} \sim d_{0} \cdot \Gamma^{n-1}$ be $\left\{C_{\zeta}^{n}\right\}$. Then $\left\{\Gamma_{\zeta}^{\prime n}\right\}=\left\{\Gamma_{\zeta}^{n}-C_{\zeta}^{n}\right\}$ is such that $F \Gamma_{\zeta}^{\prime n}=d_{0} \cdot \Gamma_{\zeta}^{n-1}$. By construction, $\mathrm{KI}\left(\Gamma_{\zeta}^{\prime n} \cdot \gamma_{n}^{\zeta}\right)=\mathrm{KI}\left(\Gamma_{\zeta}^{n} \cdot \gamma_{n}^{\zeta}\right)$, since none of the chains $C_{\zeta}^{n}$ meet $\bar{Y}$.

$\left\{\Gamma_{\zeta}^{\prime n}\right\}$ is not necessarily a Čech cycle. But, for each covering $U_{\zeta}$, let $U_{\rho(\zeta)}$ be an essential refinement (see $\left[3\right.$, II 27:13]) of $U_{\zeta}$ relative to cycles of $J^{+} \bmod \left(d_{0} \cdot H\right)^{+}$. Then $\left\{\Gamma_{\zeta}^{\prime \prime n}\right\}=\left\{\pi_{\rho(\zeta)}^{\zeta} \Gamma_{\rho(\zeta)}^{\prime n}\right\}$ is a Cech cycle $\bmod \left(d_{0} \cdot H\right)^{+}$and $\mathrm{KI}\left(\Gamma_{\zeta}^{\prime \prime n} \cdot \gamma_{n}^{\zeta}\right)=\mathrm{KI}\left(\Gamma_{\zeta}^{\prime n} \cdot \gamma_{n}\right)$ for all $\zeta$.

But now we have reached a contradiction. For $\gamma_{n} \sim 0$ in $J-d_{0} \cdot H$, so its Kronecker index with any infinite $n$-cycle of $J-d_{0} \cdot H$ must be zero. But $\mathrm{KI}\left(\Gamma^{\prime \prime n} \cdot \gamma_{n}\right)=\mathrm{KI}\left(\Gamma^{n} \cdot \gamma_{n}\right)=1$.

LEMMA 7. For each point $h \in H, r^{k}(J-H, h)=0$ for $1 \leqq k \leqq n-1$ and $r^{0}(J-H, h)=1$.

Proof. It is sufficient to consider the case $h=e$. Given any neighborhood $V$ of $e$, choose a neighborhood $V_{1}$ such that $\mathcal{C}\left(\mathcal{C}\left(V_{1} \cdot V_{1}\right)\right.$ - $B(V))$ does not meet $\bar{V}_{1}$, where $B(V)$ is the boundary of $V$. Next choose a neighborhood $V_{2}$ such that if $\gamma^{0} \subset V_{2}$, then $\gamma^{0} \sim 0$ in $V_{1}$. Let $V_{3}$ be such that $\bar{V}_{3} \cdot H$ does not contain all of $V_{2}$, and, finally, let $W$ be such that if $\gamma^{k} \subset W$, then $\gamma^{k} \sim 0$ in $V_{3}$. We assert that for $k \geqq 1$, any $\gamma^{k}$ in $W-H$ bounds in $V-H$.

For let $A$ and $B$ be the two components of $J-H$ and let $\gamma^{k}=\gamma_{a}^{k}+\gamma_{b}^{k}$, where $\gamma_{a}^{\boldsymbol{k}}$ is the part of $\gamma^{k}$ in $A$. Since $k \geqq 1, \gamma_{a}^{\boldsymbol{k}}$ is a cycle and it is sufficient to show that $\gamma_{a}^{k} \sim 0$ in $V \cap A$. If it does not, let $O$ be an open set in $W \cap A$ such that $\gamma^{k}$ is in $O$ and $O$ does not meet $H$. Then, by Lemma 5.2 of [2], there is a compact cocycle $\gamma_{n-k}$ in $O$ such that $\bar{\Gamma}^{n} \cdot \gamma_{n-k} \sim \gamma^{k}$ in $O, \gamma_{n-k}$ not $\sim 0$ in $V \cap A$, and $\gamma_{n-k} \sim 0$ in $V_{3}$. Let $\Gamma^{n-k}$ be an infinite cycle of $V \cap A$ such that $\mathrm{KI}\left(\Gamma^{n-k} \cdot \gamma_{n-k}\right)=1$.

In order to apply an argument similar to that of the preceding lemma, we choose a point of $B$ in the following fashion. Let $c$ be a point of $B$ in $V_{2}$ and not in $\mathcal{C}\left(V_{3}, H\right)$. By the choice of $V_{2}$, there is a continuum $K$ in $V_{1}$ which contains both $c$ and $e$. Let $M$ be a neighborhood of $e$ such that $M \cdot H$ does not meet $\bar{O}$. Choose $N$ by Lemma 1 and so that $N \cdot K$ is in $V_{1} \cdot N \cdot K-H$ is an open subset of $J$ and hence is locally connected. Consequently, each component of $N \cdot K-H$ is also open. Let $C$ be that component which contains $c$. Since $C$ is open and $N \cdot K$ is connected, some point $h$ in $H$ must be a limit 
point of $C . h$ is in $\bar{V}_{1}$, since $N \cdot K$ is in $V_{1}$, and therefore $D=C \cdot h^{-1}$, containing $d=c h^{-1}$, is an open connected subset of $B$ and $e$ is a limit point of $D$. Also, since $c$ is not in $V_{3} \cdot H$, neither is $d$.

From $N \cdot D$ and $N$ itself a simple chain of regions running from $e$ to $d$ can be extracted, each element of the chain being a translation of $N$.

Returning now to $\Gamma^{n-k}$, let $\Gamma^{n-k-1}$ be the part of $F \Gamma^{n-k}$ on $H$, so that $\Gamma^{n-k-1}$ is a cycle of $H \bmod X$, where $X=\bar{V} \cap \mathcal{C}(H-V)$. Using the simple chain above, we have $\Gamma^{n-k-1} \sim d \cdot \Gamma^{n-k-1}$ in $M \cdot D$. Let the chains of this homology be $\left\{C_{\zeta}^{n-k}\right\}$. Then $\Gamma_{\zeta}^{n-k}-C_{\zeta}^{n-k}$, for each $\zeta$, is, by the choice of $V_{1}$, an infinite cycle of $V_{3}$. Also, by the choice of $M$, no $C_{\zeta}^{n-k}$ meets $\bar{O}$, so $\operatorname{KI}\left(\left(\Gamma_{\zeta}^{n-k}-C_{\zeta}^{n-k}\right) \cdot \gamma_{n-k}^{\zeta}\right)=\operatorname{KI}\left(\Gamma_{\zeta}^{n-k} \cdot \gamma_{n-k}^{\zeta}\right)=1$ for each $\zeta$.

Now we can proceed to the same contradiction we reached in the previous lemma, since $\gamma_{n-k} \sim 0$ in $V_{3}$ so its Kronecker index with any infinite cycle of $V_{3}$ is zero. This disposes of the case $k \geqq 1$.

For $k=0$, let $\bar{\gamma}^{0}$ be based on a pair of points, one in $W \cap A$ and the other in $W \cap B$. The proof used above applies to show that any $\gamma^{0}$ in $W-H$ is homologous in $V-H$ to a multiple of $\bar{\gamma}^{0}$.

LEMMA 8. For each point $h$ of $H, r_{k}(h)=0$ for $k<n-1$ and $r_{n-1}(h)=1$.

This is an immediate consequence of Theorem 6.2 of [2] and Lemma 7.

LEMMA 9. $H$ is $l c^{n-1}$.

Proof. Given a neighborhood $V$ of $e$, choose $V_{1}$ in $V$ such that any $\gamma^{k+1}$ on $\bar{V}_{1}$ bounds in $V$. Choose $W \subset V_{1}$ by Lemma 7 so that any $\gamma^{k}$ in $A \cap W$ bounds in $A \cap V_{1}$ and similarly for $B$. We assert that any $\gamma^{k}$ in $W \cap H$ bounds on $\bar{V} \cap H$.

To show this it is enough to show that for any neighborhood $O$ of $e, \gamma^{k} \sim 0$ in $(O \cdot H) \cap \bar{V}$. In turn, to prove this it is sufficient to show that given any such $\gamma^{k}$ and $O$, and given any covering $U_{1}$, then there is a refinement $U_{2}$ such that $\pi_{2}^{1} \gamma_{2}^{k} \sim 0$ in $(O \cdot H) \cap \bar{V}$.

By Lemma 1 we can choose a point $a \in A \cap O$ such that $\gamma^{k} \sim a \cdot \gamma^{k}$ in $O \cdot(W \cap H)$ and we can choose a similar point $b$ in $B \cap O$. By the choice of $W, a \cdot \gamma^{k} \sim 0$ in $A \cap V_{1}$, and similarly for $b \cdot \gamma^{k}$. Thus, we have families of chains $\left\{C_{a, 5}^{k+1}\right\}$ and $\left\{C_{b, 5}^{k+1}\right\}$ in $O \cdot(W \cap H),\left\{D_{a, 5}^{k+1}\right\}$ in $A \cap V_{1}$ and $\left\{D_{b, 5}^{k+1}\right\}$ in $B \cap V_{1}$ such that

$$
\begin{array}{ll}
F C_{a, \zeta}^{k+1}=a \cdot \gamma_{\zeta}^{k}-\gamma_{\zeta}^{k}, & F C_{b, \zeta}^{k+1}=b \cdot \gamma_{\zeta}^{k}-\gamma_{\zeta}^{k}, \\
F D_{a, \zeta}^{k+1}=a \cdot \gamma_{\zeta}^{k}, & F D_{b, \zeta}^{k+1}=b \cdot \gamma_{\zeta} .
\end{array}
$$

Hence, for each $\zeta, D_{a, \zeta}^{k+1}-C_{a, \zeta}^{k+1}+C_{b, \zeta}^{k+1}-D_{b, \zeta}^{k+1}$ is a cycle $\delta_{\zeta}^{k+1}$ on $U_{\zeta}$ in 
$V_{1}$. There is a refinement $U_{2}$ of $U_{1}$ such that $\pi_{2}^{1} \delta_{2}^{k+1}$ is the coordinate of a Cech cycle, $\delta^{k+1}$ on $\bar{V}_{1}$. By the choice of $V_{1}, \delta^{k+1} \sim 0$ in $V$, so there is a chain $E^{k+2}$ on $U_{1}$ such that

$$
F E^{k+2}=\stackrel{1}{\pi_{2} \delta_{2}^{k+2}} \text {. }
$$

Let $E^{k+2}=E_{a}^{k+2}+E_{b}^{k+2}$, where $E_{b}^{k+2}$ is the part of $E^{k+2}$ on $\bar{B}$. Now

$$
F E_{a}^{k+2}-\stackrel{1}{\pi_{2}} D_{a, 2}^{k+1}=-C_{a, 2}^{k+1}+C_{b, 2}^{k+1}-D_{b, 2}^{k+1}-F E_{b}^{k+2} .
$$

The chain on the right-hand side is in $O \cdot B$ while that on the left is on $\bar{A}$. Hence, since $\bar{A} \cap \bar{B}=H, E^{k+1}=F E_{a}^{k+2}-\pi_{2}^{1} D_{a, 2}^{k+1}$ is in $O \cdot H$ and, of course, in $V$. But

$$
F\left(-E^{k+1}\right)-F\left(\pi_{2}^{1} D_{a, 2}^{k+1}\right)=\stackrel{1}{\pi_{2}} a \cdot \gamma_{a}^{k} .
$$

Hence, $\pi_{2}^{1} a \cdot \gamma_{2}^{k} \sim 0$ in $(O \cdot H) \cap V$. But $a \cdot \gamma_{2}^{k} \sim \gamma_{2}^{k}$ in $O \cdot(W \cap H)$, so $\pi_{2}^{1} \gamma_{2}^{k} \sim 0$ in $(O \cdot H) \cap V$.

At this point, we have shown, by Lemmas 8 and 9, that $H$ has the local properties of a generalized manifold. To complete the proof it only remains to show that $H$ is orientable, that is, that it carries an $(n-1)$-cycle which is not carried by any proper closed subset of $H$.

By Lemma 8, there are neighborhoods $O_{1}$ and $O_{2}$ of $e$ such that there is an $(n-1)$-cycle $\bmod H-O_{1}$ which does not bound $\bmod H-O_{2}$. By group translation, every point of $H$ has associated with it such a non-bounding relative $(n-1)$-cycle. Now an argument due to Smith [5] shows that we can carry through in the present situation the proof of Theorem 7.1 of [1] to obtain the desired $(n-1)$-cycle.

In conclusion, we point out that by restricting $G$, we can lighten the hypothesis on $H$.

TheORem. Let $G$ be a locally compact separable metric topological group which is also an orientable n-dimensional generalized manifold. Let $H$ be a closed connected ( $n-1)$-dimensional subgroup. Then $H$ is an orientable generalized manifold if any one of the following conditions is satisfied:

(1) $H$ separates some open set of $G$.

(2) For some open set $O$ of $H$, there is a nonbounding $(n-1)$-cycle of $H \bmod H-O$.

(3) $G$ is locally euclidean.

The Pontrjagin duality theorem for case (3) and Theorem 6.5 of [2] for case (2) show that both (3) and (2) imply (1). Now the proof of Lemma 1 of [4] shows that (1) yields a neighborhood of $H$ which 
is separated by $H$, that is, our Lemma 4 . Since this is the only place in our proof where the original hypothesis on $H$ is used, the rest of the proof can remain unchanged.

In case (3), if $\operatorname{dim} G=3$, we have Montgomery's theorem, for any 2-dimensional generalized manifold is locally euclidean [8].

\section{BIBLIOGRAPHY}

1. E. G. Begle, Locally connected spaces and generalized manifolds, Amer. J. Math. vol. 64 (1942) pp. 553-574.

2. - Duality theorems for generalized manifolds, Amer. J. Math. vol. 67 (1945) pp. 59-70.

3. S. Lefschetz, Algebraic topology, Amer. Math. Soc. Colloquium Publications, vol. 27, New York, 1942.

4. D. Montgomery, Analytic parameters in three dimensional groups, Ann. of Math. vol. 49 (1948) pp. 118-131.

5. P. Smith, Properties of group manifolds, Proc. Nat. Acad. Sci. U.S.A. vol. 17 (1931) pp. 674-675.

6. R. L. Wilder, Concerning a problem of K. Borsuk, Fund. Math. vol. 21 (1933) pp. 156-167.

7. - On free subsets of $E_{n}$, Fund. Math. vol. 25 (1935) pp. 200-208.

8. - Generalized closed manifolds in n-space, Ann. of Math. vol. 35 (1934) pp. 876-903.

YALE UNIVERSITY 